Research Article

\title{
Design of a Centrifugal Force-Based Speed Sensor for Downhole Turbodrills
}

\author{
Chuan Wu $\mathbb{D}^{D}$, Huafeng Ding $\mathbb{D}$, and Lei Han \\ Faculty of Mechanical and Electronic Information, China University of Geosciences (Wuhan), Wuhan 430074, China
}

Correspondence should be addressed to Huafeng Ding; dhf@ysu.edu.cn

Received 16 November 2017; Revised 19 March 2018; Accepted 3 April 2018; Published 13 May 2018

Academic Editor: Armando Ricciardi

Copyright (c) 2018 Chuan Wu et al. This is an open access article distributed under the Creative Commons Attribution License, which permits unrestricted use, distribution, and reproduction in any medium, provided the original work is properly cited.

\begin{abstract}
Turbodrill is an important type of drilling tool. Rotating speed is a key parameter to measure and control the efficiency of a turbodrill. Therefore, it is necessary to measure the rotating speed when a turbodrill is working under a well. However, current speed measurement devices and sensors cannot meet the requirements of the on-site downhole conditions due to many factors, including inadequate sealing, signal interference, difficult installation, and theoretical limitations. To break the aforementioned bottleneck, a new speed sensor used for turbodrills is designed based on the centrifugal force theory. In more detail, the measurement of the speed is performed based on a functional relation between the rotating speed and the centrifugal force of the turbodrill. Besides, the sensor was tested both in-lab and on-site. The test results illustrate that the measurement error is within $\pm 4 \%$, which meets the practical on-site conditions.
\end{abstract}

\section{Introduction}

In the wake of the rapid development of Chinese economy and society, the demand and consumption of underground mineral resources is increasing, which aggravates the contradiction between supply and demand of mineral resources. This has become a bottleneck for development of Chinese economics. In China, most shallow mineral reservoirs have already been prospected and are becoming exhausted. However, researches proved that there are abundant deep mineral resources under the ground in mainland China. Deep ore exploration has been greatly supported by Chinese government and has become a national strategy of the country [1].

A typical process of deep ore exploration is typically a two-step process. Firstly, using a drilling tool to drill downwards and taking out rocks at different depths to the ground, mineral constituents of the rocks will then be analyzed. After that, information of these constituents and their distributions and contents can be obtained via model calculation. This shows that drilling is the basis of deep ore exploration.
Figure 1 is the sketch map of the drilling process. Generally, a drilling system is constructed by a drilling rig, drill pipes, a drill bit, and a mud pump. Specifically, the drilling rig on the ground is used to hold the drill pipes and supply them with both the rotary power and the downward pressure. The drill pipes can be used with multiple connection. At the end of the drill pipes is a drill bit driven by the rotary power and the downward pressure, under which the rocks are crushed. The mud pump is used to provide power for the fluid circulation inside the borehole (Figure 1 shows the flow path and direction), so that the crushed rocks along with the mud can be taken up to the ground and filtered, which keeps the drilling process continuously working.

As the borehole goes deeper, forces acting on the drill bit and the drill pipes get more complex. Under this complex resultant force, it is impossible to keep the borehole's track vertical, which makes the track itself more complicated. As a result, the friction between the drill pipes and the borehole becomes larger. Especially for deep drilling, the friction is larger due to the long distance from the ground to the bottom of the borehole. Consequently, it is difficult to transfer the power from the drilling rig 


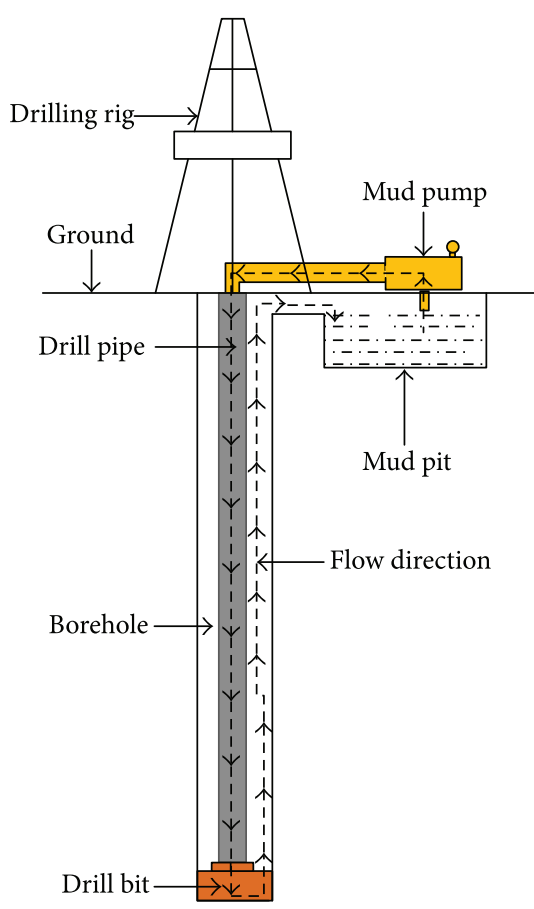

FIGURE 1: Sketch map of the drilling process.

to the drill bit, which indeed decreases the drilling efficiency. Besides, there is a possibility that the large friction might fracture the drill pipes and cause an accident in consequence [2]. To secure the drilling and raise the efficiency, downhole drilling motors have been commonly used for deep drilling recently. For more details, the downhole drilling motor at the bottom of the borehole is driven by the high pressure of fluid from the mud pump on the ground. Therefore, the rocks at the bottom of the borehole can be crushed by the drill bit connected to the motor.

Common downhole drilling motors include a downhole hammer, screw motor, and turbodrill that are widely used and are applicable for deep and high-temperature wells [3].

Figure 2 is the sketch map of the turbodrill and its working principle, as shown in Figures 2(a) and 2(b), respectively. Figure 2 (a) shows that the turbodrill contains a turbine section and a bearing section. A number of turbine blades are mounted on the turbine section. Figure 2(b) shows that the top of the turbine section is connected with the drill pipe while the bottom of the bearing section (containing a gear reducer) is connected with the drill bit. When the turbodrill is working, the mud (a chemically prepared solution) is pumped out from the mud pit and enters into the turbodrill through the drill pipes (the flow direction is shown in Figure 2(b)). In this case, the blades start to rotate under the impact of the fluid, and the rotation is further delivered to the drill bit via the bearing section. Then, the rotating drill bit grinds the rocks at the bottom of the borehole and, therefore, realizes the purpose of drilling [4,5]. At this moment, the mud flows out of the drill bit and takes the crushed rocks back to the mud pit through the annular gap between the borehole and the drill pipes.
For a turbodrill, rotating speed and torque are the most important indicators of the turbodrill. When the rotating speed and torque are appropriate, the efficiency of the turbodrill is highest. To date, there have been no suitable measurement methods for downhole torque, which implies that the efficiency can only be judged by the rotating speed measurement of downhole speed. Therefore, it is necessary to measure the rotating speed when it is working under a well. By now, methods of measuring turbodrill rotating speed include magneto-electric speed measurement, photoelectric speed measurement, flow deduction method, and pressure pulse method. However, these methods are not fully applicable in downhole conditions due to some limitations as follows.

(1) Magneto-electric speed measurement: based on the law of electromagnetic induction [6], the measurement can be implemented by using a magnetoelectric speed sensor that is placed on the shell of the turbine section (when it is in a static state). After being powered on, the sensor generates magnetic induction lines that are cut by the rotating bearing section, which generates the induced electromotive force in the sensor. Therefore, the rotating speed can be measured based on the fact that the speed is directly in proportion to the induced electromotive force.

Magneto-electric speed measurement has the following disadvantages that limit its applications to the turbodrill in the downhole conditions:

(a) Inadequate sealing: magneto-electric speed sensor cannot be applied in the case of high pressure in a well due to the lack of high-level sealing. If the sensor is used in a well, one has to design the sealing structure. In this case, if the sealing is too thick, the magnetic induction lines generated by the sensor may be shielded; if the sealing is too thin, it may be squashed off by the high-pressure water, which will result in the failure of the sensor.

(b) Difficult installation: the sensor has to be mounted on the turbine section to take the measurement when the bearing section is cutting the magnetic induction line generated by the sensor. The measurement is not feasible if the sensor is mounted at a relatively remote place, because the strength of the lines is too weak. However, the installation point cannot be precisely ensured because of the complex internal structure of the turbodrill.

(c) Signal interference: the bearing section of the turbodrill is made of a metal material so that it can generate the induced electromotive force by cutting the magnetic induction lines. However, the turbine section and other parts are also made of metal material, which will cause interference to the sensor when moving.

(2) Photoelectric speed measurement: the measurement is realized by a photoelectric speed sensor, which comprises a transmitter and a receiver. The 


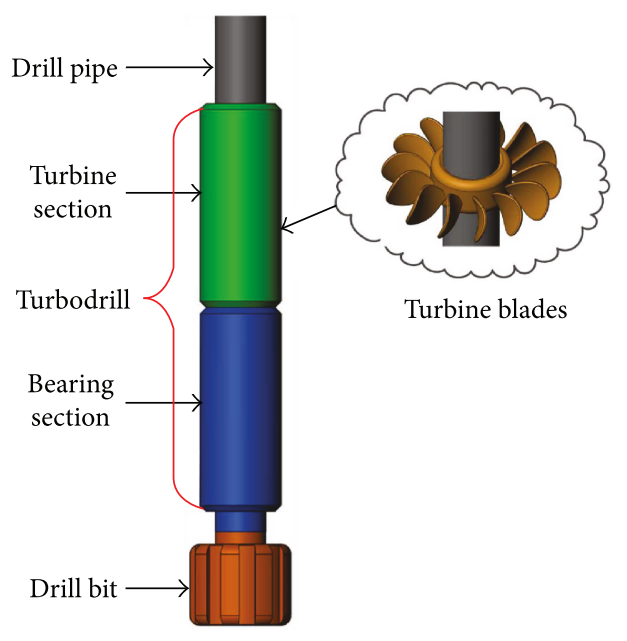

(a)

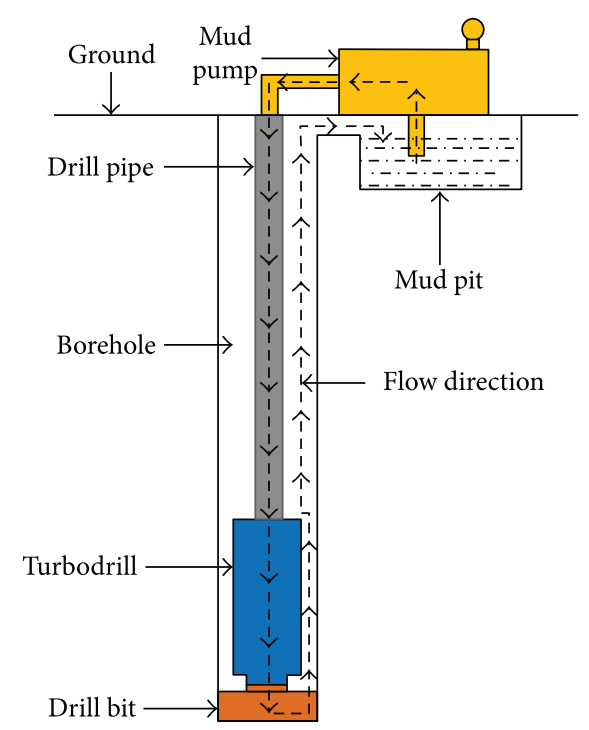

(b)

FIGURE 2: Sketch map of the turbodrill and its working principle.

transmitter is fixed while the receiver rotates along with the object to be measured. The transmitter transmits a light signal at a certain power, and the receiver receives the light signal every time it rotates a circle. Then, the rotating speed can be determined according to the frequency of receiving the light signal [7]. Photoelectric speed measurement is not applicable under downhole conditions because of the mud existing in the environment where the turbodrill works. The mud is a kind of turbid solution, and the degree of the turbid has a direct effect on the reception to the light signal. This is why this method is not applicable under downhole conditions.

(3) Flow deduction method: in this method, the measurement is realized based on the functional relation between flow rate of the fluid and rotating speed of the turbodrill [8]. By the working principle of the turbodrill, the rotation of the turbodrill is driven by the mud pumped through the drill pipes. Theoretically, therefore, the speed is larger if the flow rate of the mud is higher. This relation is the basis of the measurement for the rotating speed. The key of this method is the measurement of the flow rate. There are two flow rates that can be measured: the flow rate from the ground to the borehole and that from the bottom of the borehole to the turbodrill. For this method, it is obvious that the flow rate from the bottom of the borehole to the turbodrill is needed to be measured. By far, the sealing of the flow sensor cannot fully meet the requirements of applications in the downhole conditions. So, it is not feasible to measure the flow rate at the bottom of the borehole. In the case that the measurement of the mud flow rate is performed on the ground, however, it would result in a large error because of the following reasons. In theory, the two flow rates are equivalent to each other. But in practical, there is a linear flow loss along the long distance from the mouth to the bottom of the borehole, and this flow loss cannot be precisely calculated. Moreover, the two flow rates are not equivalent to each other because of water gushing (from the stratum to the borehole) or seepage (from the borehole to the stratum). In spite of the big, random, and incalculable error, this method has still been widely used because there are no other precise methods available to measure the downhole rotating speed.

(4) Pressure pulse method: in this method, a speed transmitter is mounted on the turbodrill. The transmitter can convert the rotating speed of the turbine into a mud pulse and transmit the pulse up to the ground through the circulation channel of the mud. The real-time pulse signals can be received by a number of pressure sensors mounted on the ground. Finally, the rotating speed of the turbine can be determined by making and filtering the spectral analysis of the received pulse signals [9].

This method has some obvious deficiencies that limit its application to the turbodrill under downhole conditions:

(a) Signal interference: in this method, the signal is transferred along with the mud pulse, which will be affected if water gushing or seepage exists in the stratum. In addition, the mud pumped out of the pit itself carries a pulse at a certain frequency that also generates the interference.

(b) Complex equipment: this method requires one to mount a specially designed speed transmitter in the borehole and a number of pressure transmitters on 
the ground. The equipment is complex, and the installation is also inconvenient.

(c) Complex data processing: this method requires a number of pressure transmitters mounted on the ground to extract the signals at the same time. Then, the pulse frequency can be obtained by observing the spectrogram with the eyes. Finally, the functional relation between the pulse frequency and the rotating speed is required to calculate the speed. This data processing method is too complex to realize real-time measurement. To sum up, the magnetoelectric speed measurement cannot be used to measure the rotating speed of the turbodrill under downhole conditions due to many limitations, including inadequate sealing, difficult installation, and signal interference. Affected by mud in the borehole, the photoelectric speed measurement generates large and random errors. Thus, this method is not applicable in the borehole but is commonly used on the ground. The flow deduction method also generates large and random errors because of both the linear flow loss and the water gushing or seepage in the stratum. The pressure pulse method is likewise inapplicable for downhole measurement due to many problems such as signal interference, complex equipment, and complex data processing. Limited to special downhole conditions, the above methods cannot meet the requirement for measuring the rotating speed of the turbodrill in a borehole. Given this, this paper has purposely designed a centrifugal force-based speed sensor that can be used to measure the rotating speed of the downhole turbodrill by using the functional relation between the centrifugal force and the rotating speed. Compared with the above methods, this sensor is more applicable under downhole conditions and can reduce the measurement error and, theoretically, raise the measurement accuracy greatly.

\section{Measurement Indexes}

Prior to the design, we have to designate several indexes for the measurement and work out the entire scheme of the sensor in accordance with these indexes. Specifically, the indexes include sealing, applicable temperature, measurement range, and electric parameters, which are given as follows.

(1) Sealing: the sensor shall be mounted at the bottom of the borehole, where the water pressure becomes bigger as the borehole gets deeper. After our site survey, we set $30 \mathrm{MPa}$ for the sealing of our sensor. It is wellknown that $1 \mathrm{MPa}$ is equivalent to 100 meters of water column. If the density of the mud equals to water density, then the sensor can be used at the maximum depth of 3000 meters.

(2) Temperature: a geothermal gradient exists in the Earth's interior. The temperature increases by about $3^{\circ} \mathrm{C}$ per 100 meters of depth [10]. Therefore, the temperature at the depth of 3000 meters is about $90^{\circ} \mathrm{C}$ higher than that on the ground (the temperature at the bottom of the borehole can be greatly decreased under the cooling effect of the mud circulation). Thus, we set $125^{\circ} \mathrm{C}$ as the maximum applicable temperature of the sensor.

(3) Measurement range: after literature review [11] and site survey, we determine that the range of the rotating speed of the turbodrill with a gear reducer is 55$200 \mathrm{r} / \mathrm{min}$.

Table 1 is the summary of the basic performance indexes for designing the speed sensor.

\section{Sensor Design}

3.1. Basic Principle. Figure 3(a) shows the basic configuration of the sensor, which consists of a shaft, a probe, a shell, and a circuit board. The probe is used to realize real-time measurement of the rotating speed. The measurement data is simultaneously transferred to the circuit board for analysis, processing, and storage. The shell is used to seal the probe and the circuit board. The shaft that has through-holes for mud flowing is connected to the turbodrill.

Figure 3(b) shows the internal structure of the probe, which consists of a guide bar, a mass block, a spring and a force sensor. The mass block moves along the direction shown in Figure 3(b) under the centrifugal force when the turbine rotates. The end of the mass block is linked to the spring, and the other end is linked to the force sensor. When the centrifugal force acting on the mass block is measured by the force sensor, the real-time measurement data is simultaneously inputted to the circuit board for processing and storage. When the turbine stops rotating, the centrifugal force disappears and the mass block will be pulled back by the spring.

By the sensor's structure shown in Figure 3, to use the sensor we first have to mount the probe, the circuit board, and the battery onto the shaft (the sensor itself contains a battery to support the storage of the real-time data) then start the power to collect data. Next, the shell shall be assembled to seal the shaft, whereby the start-up and installation of the sensor is completed.

The model of the selected force sensor is XFL212R (MEAS Company). The main parameters are as follows:

(i) Measurement range: $0-5 \mathrm{~N}$

(ii) Measurement accuracy: $\leq \pm 0.5 \%$

(iii) Operating temperature range: $-40^{\circ} \mathrm{C} \sim 150^{\circ} \mathrm{C}$

3.2. Key Technical Points. By the working principle of the sensor, the key technical points include the establishment of a physical model, the computation of the sensor's measurement range, and the design of the data storage circuit, which are described below. 
TABLe 1: Parameters to design speed sensor for downhole turbodrill.

\begin{tabular}{lc}
\hline Measurement range & $55 \mathrm{r} / \mathrm{min} \leq v \leq 200 \mathrm{r} / \mathrm{min}, v:$ rotating speed \\
Measurement error & $\pm 4 \%$ \\
Output signal & Digital signal output \\
Supply voltage & $\mathrm{DC} 5 \mathrm{~V}$ \\
Applicable pressure & $0-30 \mathrm{MPa}$ \\
Applicable temperature & $0-125^{\circ} \mathrm{C}$ \\
Sampling frequency & $10 \mathrm{~Hz}$ \\
Data storage period & 4 days (the sensor has the function of storage)
\end{tabular}

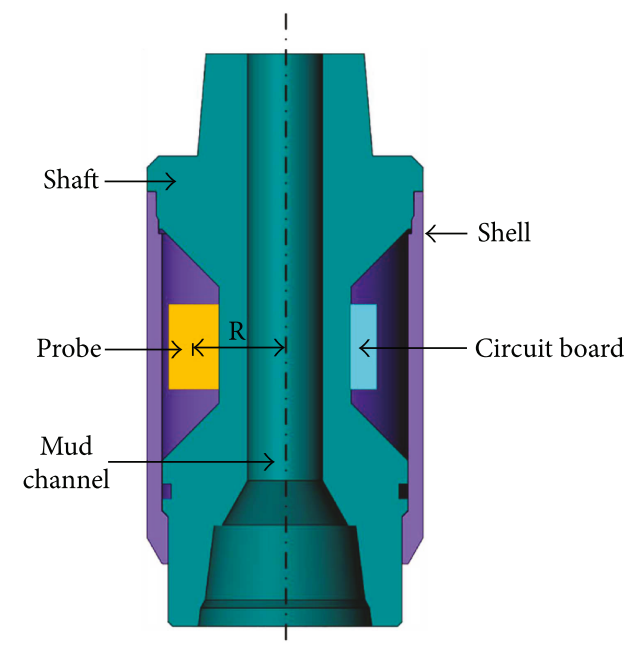

(a)

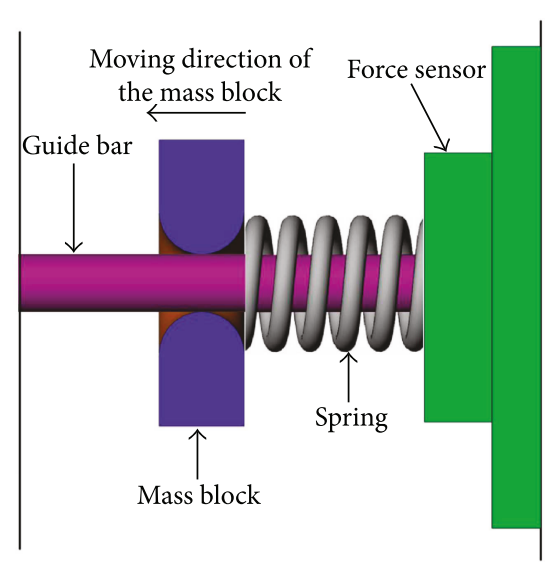

(b)

FIGURE 3: Sketch map of the sensor's configuration.

3.2.1. Physical Model. The physical model of the mass block (in Figure $3(b)$ ) is built when the sensor is working. Figure 4 shows the mechanical model of the mass block. By analyzing the mechanical model, we can obtain the following equations:

$$
\begin{gathered}
G=N, \\
F=f+F_{1}, \\
G=m g, \\
f=\mu N, \\
F=\frac{m v^{2}}{R}, \\
F_{1}=k \cdot \Delta R_{1}, \\
R=R_{0}+R_{1}+\Delta R_{1}+R_{2},
\end{gathered}
$$

where $G$ is the gravity, $N$ is the supporting force, $F$ is the centrifugal force, $f$ is the friction, $F_{1}$ is the spring tension, which can be measured by the force sensor shown in Figure 3(b), $m$ is the mass of the mass block, $g$ is the gravity constant, $\mu$ is the friction coefficient, $v$ is the turbine speed, $R$ is the distance from the barycenter of the mass block to the axis of the sensor, $R_{0}$ is the distance from the barycenter of the mass block to the long edge, $R_{1}$ is the original length of the spring, $\Delta R_{1}$ is the extension of the spring, $R_{2}$ is the distance from the axis of the sensor to the spring, and $k$ is the elastic coefficient of the spring.

From (1), (2), (3), (4), (5), (6), and (7), we can conclude that

$$
v=\sqrt{\left(\mu g+\frac{F_{1}}{m}\right)\left(R_{0}+R_{1} \frac{F_{1}}{k}+R_{2}\right)} .
$$

Equation (8) implies that we neglect the mass of the spring. The reasons are given as follows.

According to our design idea, the spring is not in contact with the guide bar; that is, the spring is supported by the mass block and the force sensor. Assuming that the mass of the spring is $m_{1}$, the mass of the spring section supported by the mass block is $m_{2}$, and the mass of the spring section supported by the force sensor is $m_{3}$. By analyzing the mechanical model of Figure 4, we can conclude that

$$
\begin{array}{r}
m_{1}=m_{2}+m_{3}, \\
f=\mu g\left(m+m_{2}\right),
\end{array}
$$




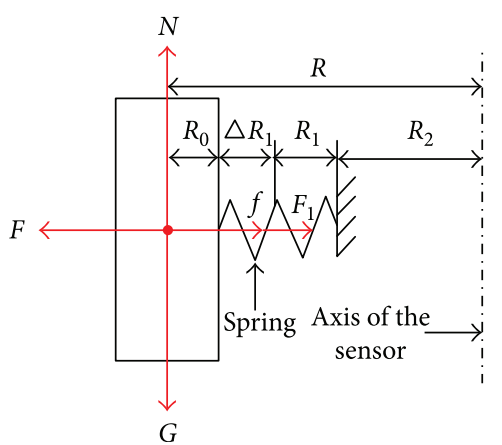

Figure 4: Mechanical model of the mass block.

where $f$ is the friction, $\mu$ is the friction coefficient, $g$ is the gravity constant, $m$ is the mass of the mass block, $m_{1}$ is the mass of the spring, $m_{2}$ is the mass of the spring supported by the mass block, and $m_{3}$ is the mass of the spring supported by the force sensor.

In ideal conditions, $m_{2}$ is equal to $m_{3}$. They are not equal with reality because of the complex stress conditions. So we cannot obtain the exact solution of (10). Moreover, the mass of the spring is only about 1/20 of the mass of the mass block; that is, the friction is mainly produced by the mass block and the friction produced by the spring is almost negligible.

Hence, we neglect the mass of the spring.

\subsubsection{Measurement Range}

(1) The detected minimum velocity $v_{\min }$ : the centrifugal force is smaller than the friction when the rotation is too slow. In this case, the mass block keeps still and the output data measured by the sensor is 0 . Hence, the minimum velocity $v_{\min }$ detected by the sensor is

$$
F \leq f \Rightarrow v_{\min }=\sqrt{\mu g\left(R_{0}+R_{1}+R_{2}\right)} .
$$

(2) The detected maximum velocity $v_{\max }$ : the centrifugal force is far larger than both the friction and the spring tension when the rotation is too fast. The spring will have irreversible plastic deformation if the tension force is beyond its bearing capacity, which will cause damage to the sensor. Hence, the maximum velocity $v_{\max }$ detected by the sensor shall be

$$
\begin{aligned}
F & \geq f+F_{1 \max } \Rightarrow v_{\max } \\
& =\sqrt{\frac{\left(\mu m g+F_{1 \max }\right)\left(R_{0}+F_{1 \max } / k+R_{1}+R_{2}\right)}{m}},
\end{aligned}
$$

where $F_{1 \max }$ is the maximum force that the spring can bear.
(3) The sensor's measurement range: it can be inferred from (11) and (12) that the sensor's measurement range $S_{a}$ is

$$
\begin{aligned}
S_{a} & =v_{\max }-v_{\min }, \\
S_{a} & =\sqrt{\left(\mu g+\frac{F_{1 \max }}{m}\right)\left(R_{0}+R_{1}+\frac{F_{1 \max }}{k}+R_{2}\right)} \\
& -\sqrt{\mu g\left(R_{0}+R_{1}+R_{2}\right)} .
\end{aligned}
$$

From (8), (9), (10), (11), (12), (13), and (14), we can know that the parameters of the sensor required to be designed include the friction coefficient $(\mu)$, the mass of the mass block $(m)$, the distance from the barycenter of the mass block to the long edge $\left(R_{0}\right)$, the original length of the spring $\left(R_{1}\right)$, the elastic coefficient of the spring $(k)$, and the distance from the axis of the sensor to the spring $\left(R_{2}\right)$. The principle of parameter selection is that the value of $v_{\min }$ should be as small as possible, while the values of $v_{\max }$ and $S_{a}$ should be as large as possible. Meanwhile, the sensor should not be oversized. When designing the sensor, we can preliminarily select the parameters in accordance with their equations. The selected parameters can then be used to compute the values of $v_{\max }, v_{\min }$, and $S_{a}$. If the computed values meet the indexes of the sensor design, the selected parameters are proper. Otherwise, we have to modify the improper ones till all of them meet the practical engineering requirements.

After many selections, the parameter values of the sensor are determined as shown in Table 2.

By substituting the values into (11), (12), and (14) with the unit of $\mathrm{r} / \mathrm{min}$, we can obtain $v_{\min } \approx 53 \mathrm{r} / \mathrm{min}, v_{\max } \approx 201$ $\mathrm{r} / \mathrm{min}$, and $S_{a}=148.7 \mathrm{r} / \mathrm{min}$.

3.2.3. Data Storage Circuit. The real-time data of the sensor are simultaneously sent to a microprocessor. After being processed in the microprocessor, the data are saved in an SD card. The mode of SPI is used as the communication protocol between the microprocessor and the SD card. The power source of the microprocessor is $5 \mathrm{~V}$ while the power source of the $\mathrm{SD}$ card is $3.3 \mathrm{~V}$. Therefore, the electrical levels for the communication shall be matched. Here, 1N4732, one type of voltage-stabilizing diode, is used to match the level. Figure 5 shows the design of the level matching circuit for the SD card.

Moreover, if the rotating speed data is monitored in real time, the wired transmission can be used under the RS485 communication protocol. The signal relay station can be placed every 1000 meters, and the baud rate must be as low as possible in order to ensure the signal is not distorted. In this way, the downhole rotating speed data can be collected by the ground control unit in real time.

3.2.4. Resolution. The resolution of the designed sensor is related to the resolutions of both the sensor in Figure 3(b) and the DAC (digital-to-analog converter). Based on the selected chip, we determine that the sensor's resolution is $0.01 \mathrm{r} / \mathrm{min}$. 
TABLE 2: Parameter values of the sensor structure.

\begin{tabular}{lcc}
\hline Parameter & Value & Note \\
\hline$\mu$ & 0.15 & Pig iron-pig iron (rust prevention), no lubrication \\
$m$ & $0.0414 \mathrm{~kg}$ & Cylinder, diameter $22 \mathrm{~mm}$, thickness $15 \mathrm{~mm}$, with a $\varphi 6 \mathrm{~mm}$ through-hole \\
$R_{0}$ & $7.5 \mathrm{~mm}$ & \\
$R_{1}$ & $12 \mathrm{~mm}$ & \\
$R_{2}$ & $30 \mathrm{~mm}$ & The extreme extension amount is $25 \mathrm{~mm}$ \\
$k$ & $52.29 \mathrm{~N} / \mathrm{m}$ & \\
\hline
\end{tabular}

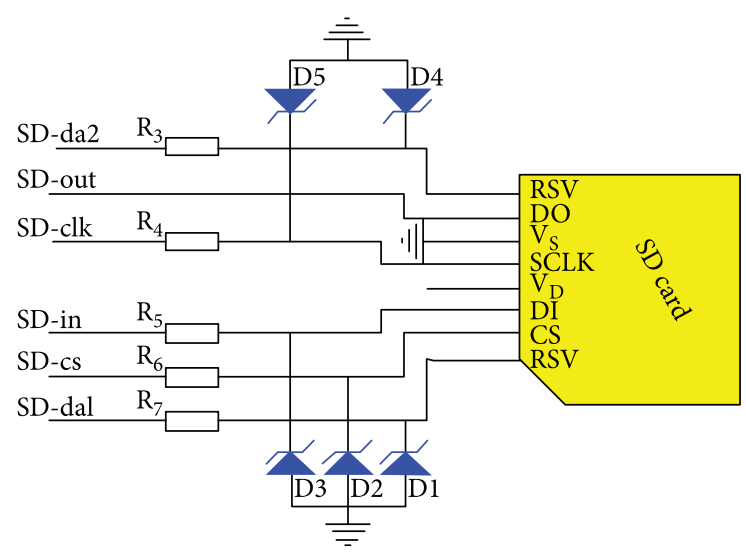

FIGURE 5: Level matching circuit.

\section{Experiment}

Experiments have been conducted both in laboratory and on-site. In the laboratory experiment, the sensor's reliability and accuracy are tested. In the on-site experiment, the sensor's adaptability to practical conditions is tested. Here are the descriptions.

4.1. Laboratory Experiment. The size of the actual sensor is too large (diameter: $240 \mathrm{~mm}$ and length: $1080 \mathrm{~mm}$ ) and heavy (weight: $300 \mathrm{~kg}$; a special hoisting equipment is required to move the sensor). For these reasons, we have modified the sensor so that it can be tested in the laboratory. The modification includes two aspects:

(1) The sensor is scaled down in proportion $(2.5: 1)$ to reduce its size and weight so that it can be easily moved and installed in the laboratory for the test.

(2) The thread of the sensor's shaft is changed so that it can be connected with the testing device in the laboratory.

4.1.1. Experiment Equipment. The laboratory testing equipment designed by ourselves is shown in Figure 6. One end of the sensor is connected with a motor which contains a gear reducer, and the other end is connected with a standard speed sensor (it also has the function of measuring torque). The motor can output different rotating speeds by the use of the gear reducer. Data of the speeds can then be collected by both our sensor and the standard sensor. In our sensor, the real-time data are stored in the SD card and can be read when the motor stops working. In the standard sensor, the real-time data are stored in an upper computer and can be simultaneously displayed. The reducer has eight gear pairs, so there are also eight velocities available for the laboratory test system, which are 47, 57, 71, 86, 107, 130, 183, and 222 (unit: $\mathrm{r} / \mathrm{min}$ ), respectively. For our sensor, the measurement range is [53,201] (unit: $\mathrm{r} / \mathrm{min}$ ), which corresponds to six velocities, namely, 57, 71, 86, 107, 130, and 183 (unit: $\mathrm{r} / \mathrm{min}$ ), respectively.

4.1.2. Experiment Environment. The experiment was carried out at room temperature and normal atmosphere.

4.1.3. Experiment Steps. The measured value and the standard value can be collected by the designed sensor and the standard sensor, respectively. The data collection time is 10 minutes for each session. Subsequently, the speed of the laboratory testing equipment is repeatedly adjusted in order to ensure that each speed is tested 200 times.

4.1.4. Measurement Errors. Make statistics of the measurement errors when the rotating speed is $57 \mathrm{r} / \mathrm{min}, 71 \mathrm{r} / \mathrm{min}$, $86 \mathrm{r} / \mathrm{min}, 107 \mathrm{r} / \mathrm{min}, 130 \mathrm{r} / \mathrm{min}$, and $183 \mathrm{r} / \mathrm{min}$, respectively. In our experiment, the sampling frequency is $10 \mathrm{~Hz}$, the period for each test is $10 \mathrm{~min}$, and the total number of the tests is 200. Thus, the number of the data corresponding to each rotating speed is 1200000 .

Figures 7 to 12 show the curves of the data collected in 50 successive tests from the data of each rotating speed, and the data contain the maximum measurement error which is represented as $\varepsilon_{\max }$. The abscissa represents the number of the test, and the ordinate represents the rotating speed.

$$
\varepsilon_{\max }=\frac{\left|S_{s}-S_{t}\right|}{S_{s}} \times 100 \%,
$$

where $S_{s}$ is the standard value of the rotating speed, which is measured by the standard sensor, and $S_{t}$ is the measured value of the rotating speed, which is measured by the designed sensor.

From analysis of the curves shown in Figures 7 to 12, we know that the maximum measurement errors are $3.97 \%$, $3.81 \%, 3.97 \%, 4 \%, 3.89 \%$, and $3.39 \%$ corresponding to the rotating speeds of $57 \mathrm{r} / \mathrm{min}, 71 \mathrm{r} / \mathrm{min}, 86 \mathrm{r} / \mathrm{min}, 107 \mathrm{r} /$ $\mathrm{min}, 130 \mathrm{r} / \mathrm{min}$, and $183 \mathrm{r} / \mathrm{min}$, respectively. Therefore, the final measurement error is the largest one: $4 \%$, which satisfies the design requirements. 


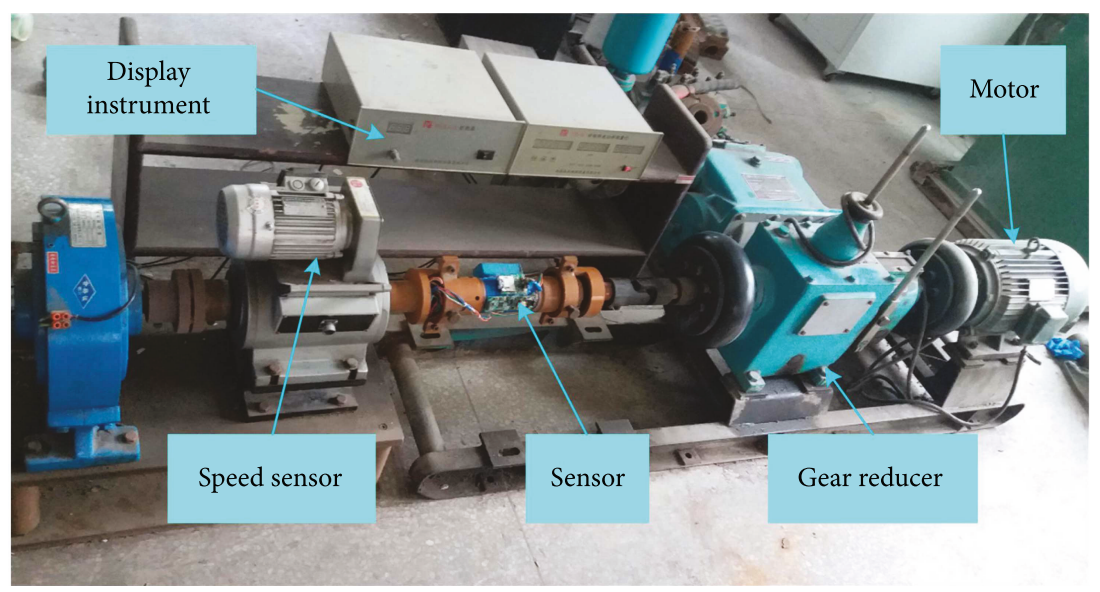

Figure 6: Laboratory testing equipment.

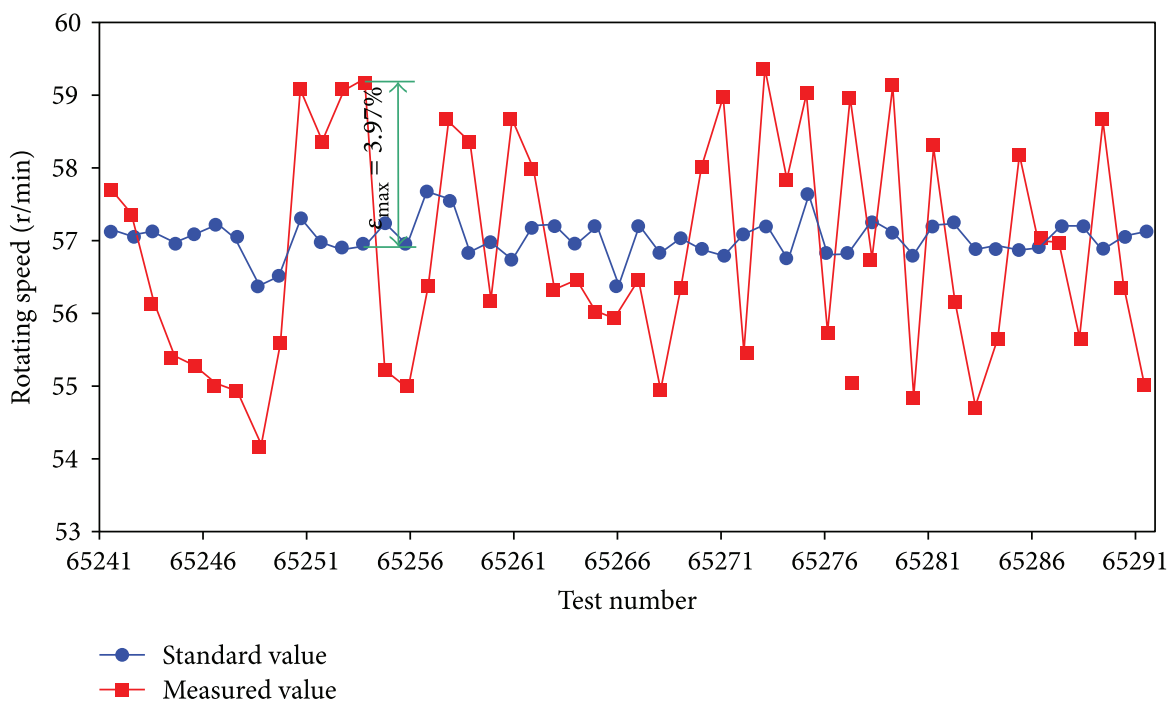

FiguRE 7: Comparing measurement performance of the proposed sensor to the standard speed sensor at the rotational speed of $57 \mathrm{r} / \mathrm{min}$.

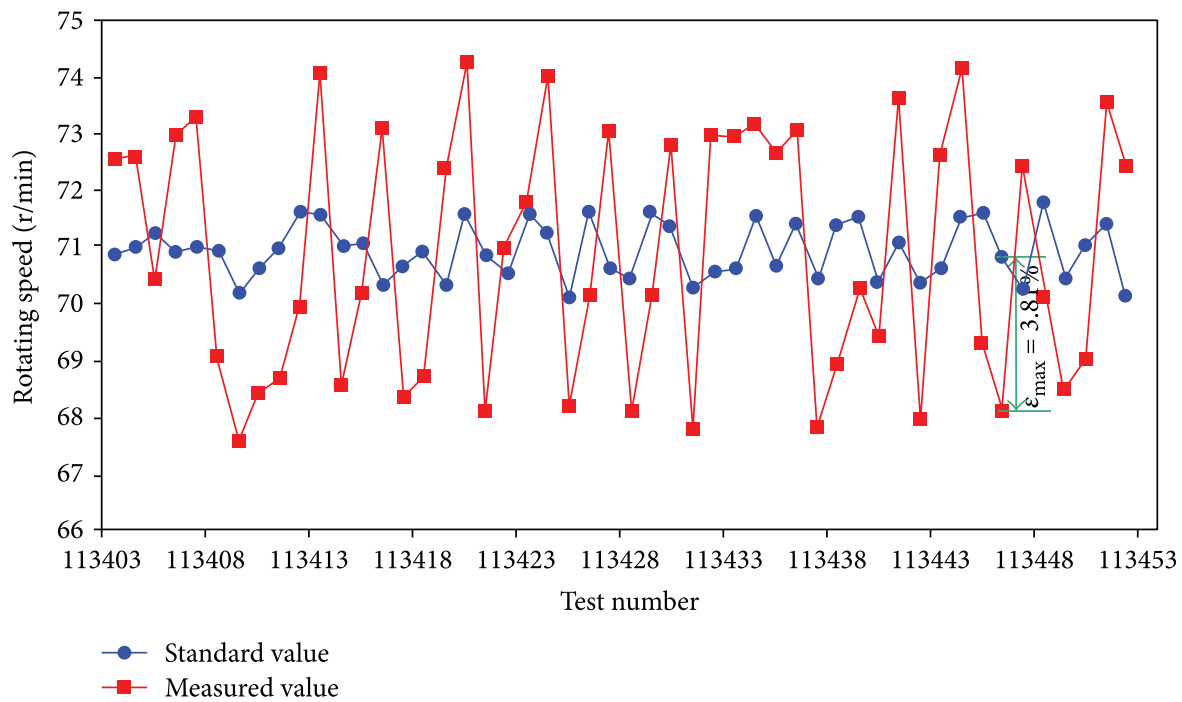

Figure 8: Comparing measurement performance of the proposed sensor to the standard speed sensor at the rotational speed of $71 \mathrm{r} / \mathrm{min}$. 


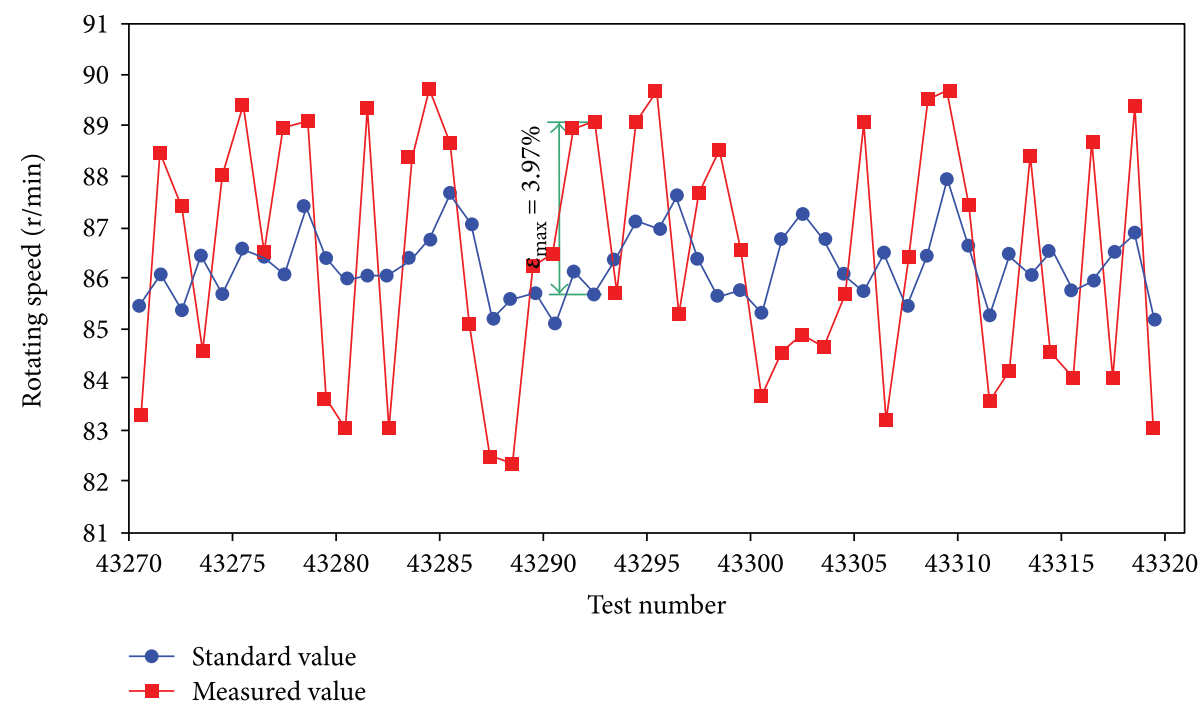

FIGURE 9: Comparing measurement performance of the proposed sensor to the standard speed sensor at the rotational speed of $86 \mathrm{r} / \mathrm{min}$.

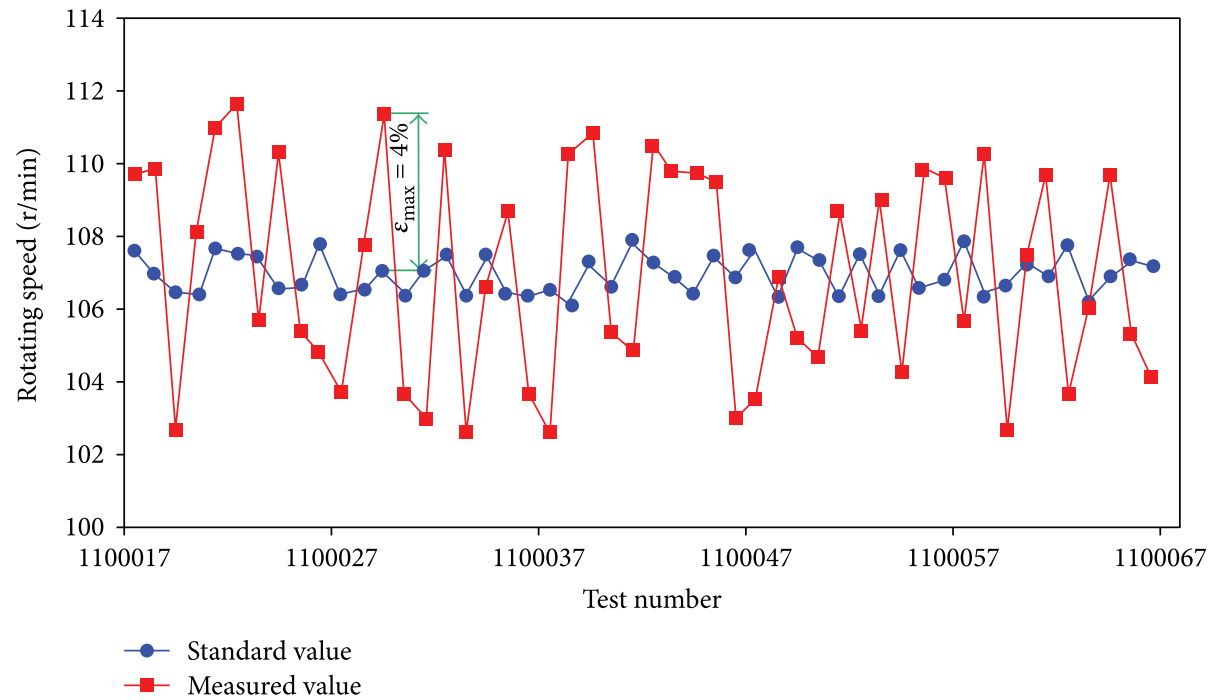

FIGURE 10: Comparing measurement performance of the proposed sensor to the standard speed sensor at the rotational speed of $107 \mathrm{r} / \mathrm{min}$.

Besides, the measurement error of the developed sensor is not accurate as compared to the standard speed sensor. The reason is that the standard speed sensor consists of electronic components while the developed sensor mainly consists of mechanical components, especially frictional drag and the microplastic deformation of the mechanical components.

4.2. On-Site Experiment. By far, there are no standard and accurate devices for measuring the rotating speed of the turbodrill in the downhole. Therefore, the on-site experiment can only test the reliability of the sensor and its adaptability to practical conditions.

4.2.1. Profile of the Test Well. The Chinese Continental Science Drilling (CCSD, a project of the International Continental Drilling Program in China) is taken as the test well, which is located at Liuzuofang village, Anda city,
Heilongjiang province, China. The depth of the well is 2875 meters, and the temperature at the bottom is $92.5^{\circ} \mathrm{C}$ (the depth and temperature of the well come from the internal logging information).

4.2.2. Installation of the Sensor. Figure 13 shows the on-site experiment of the sensor. Figure 13(a) is a picture of the sensor, and Figure 13(b) shows the downhole conditions. As shown in Figure 13(b), the upper end of the turbodrill is connected with the drill pipe, and its lower end is connected with the sensor, under which is the drill bit.

4.2.3. Experiment Process. Start the power of the sensor before assembly, and seal the sensor. Install the sensor between the turbodrill and the bit, and then place the turbodrill together with the sensor at the bottom of the borehole to collect the data. A period later (generally, the period is 3 


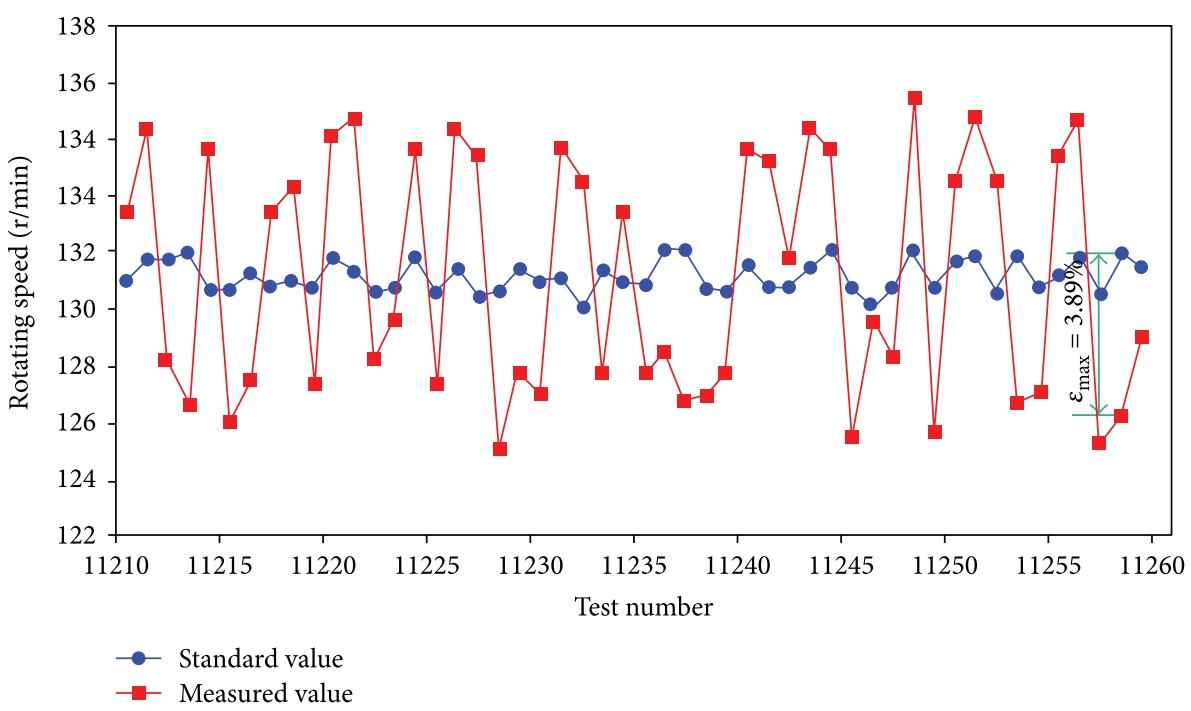

FIGURE 11: Comparing measurement performance of the proposed sensor to the standard speed sensor at the rotational speed of $130 \mathrm{r} / \mathrm{min}$.

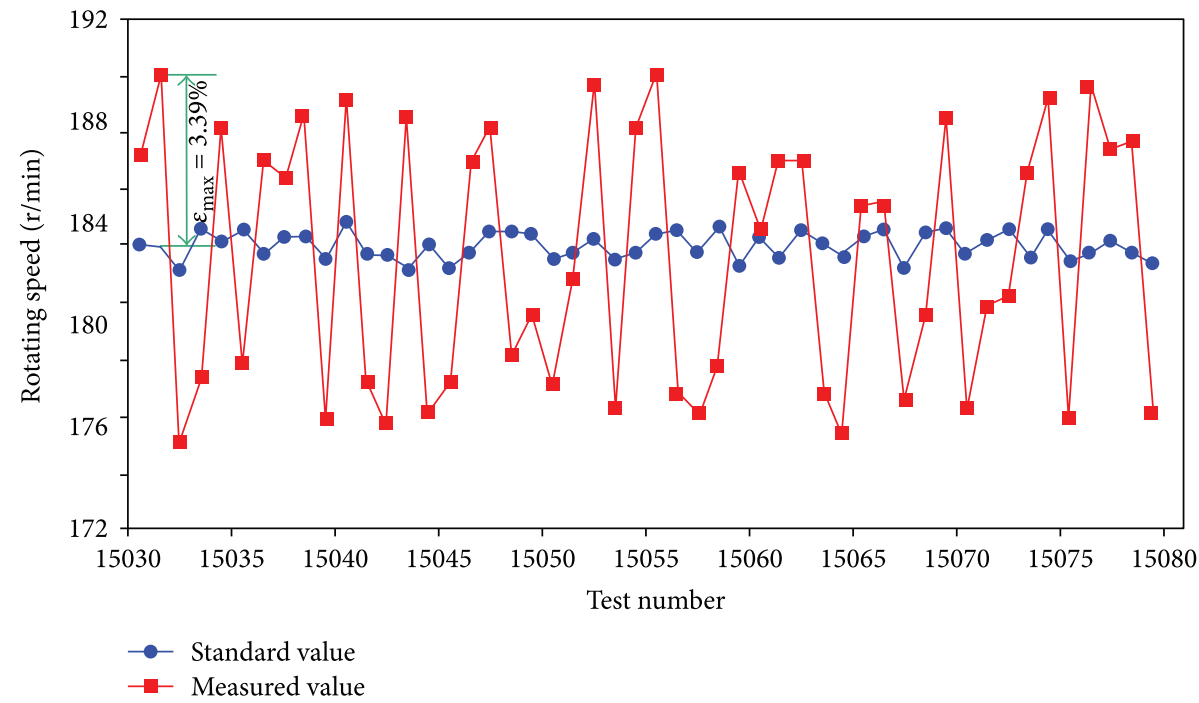

FIGURE 12: Comparing measurement performance of the proposed sensor to the standard speed sensor at the rotational speed of $183 \mathrm{r} / \mathrm{min}$.

days), use the rig to lift the turbodrill and the sensor up to the ground, and then use software to read the data stored in the sensor. In order to obtain as much test data as possible, the number of test times is needed to be as high as possible.

4.2.4. Experiment Results. The following can be concluded from one of the test curves of on-site performance as shown in Figure 14:

(1) The on-site data collected by the sensor proves that the sensor has no leakage and its sealing performance can meet the downhole conditions.

(2) From the comparison between the data of both the flow rate and the time recorded on the ground and the data collected by the sensor, we have the following information. The periods during which the flow rate keeps unchanged during the $\mathrm{t} 2$ and $\mathrm{t} 3$ phases are 2.32 and 3.77 hours, respectively. With respect to the sampling frequency of the sensor, we know that the numbers of the data theoretically collected by the sensor during $\mathrm{t} 2$ and $\mathrm{t} 3$ are 83520 and 135720, respectively. As shown in Figure 14, the numbers of the data actually collected by the sensor during $t 2$ and $t 3$ are 82241 and 133607, respectively, which are close to the theoretical case. The deviation might be caused by some complex factors such as the propagation delay and the linear loss of the flow. This, from another aspect, verifies that the data collected by the sensor are correct and can meet the practical working conditions.

(3) From the comparison between the data of both the flow rate and the time recorded on the ground and 


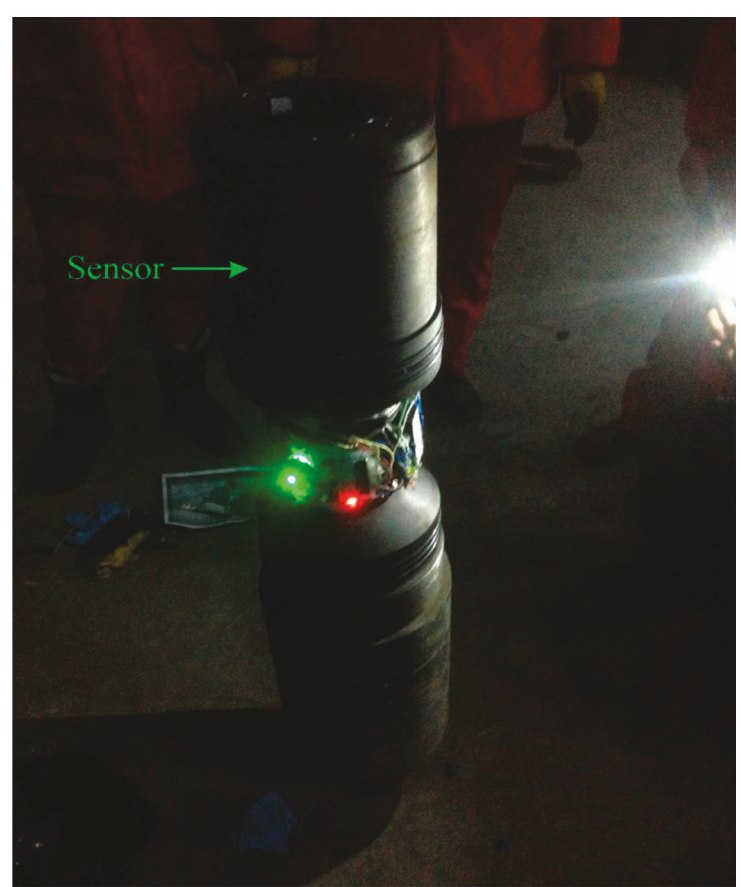

(a)

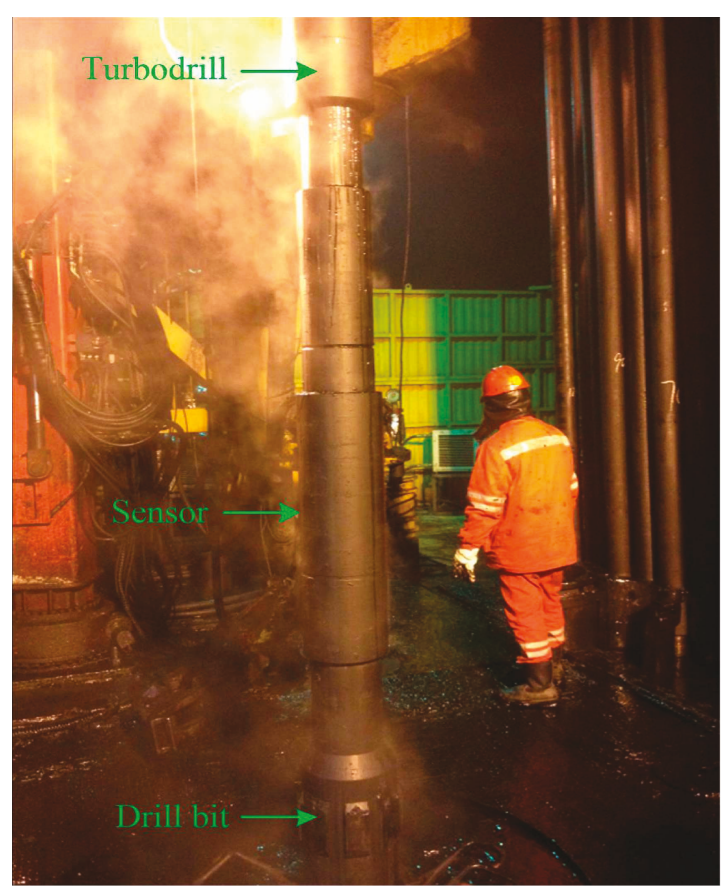

(b)

FIGURE 13: Installation position of sensor in the on-site experiment.

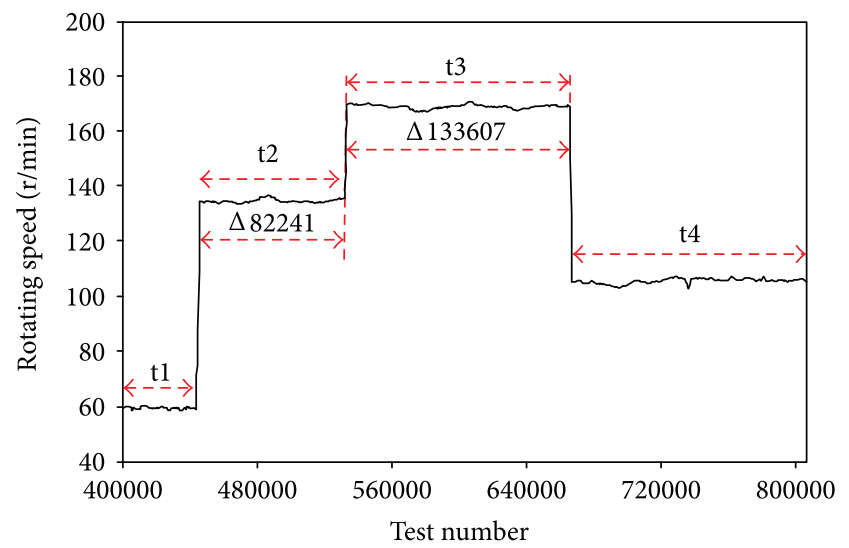

Figure 14: Test curve of on-site performance.

the data collected by the sensor, we have the following information. The flow rate increases during the period from $t 1$ to $t 3$ and decreases during the period from $\mathrm{t} 3$ to $\mathrm{t} 4$. In Figure 14, the rotating speed practically recorded by the sensor also increases during the period from $t 1$ to $t 3$ and decreases during the period from $\mathrm{t} 3$ to $\mathrm{t} 4$. In other words, the variation trend of the rotating speed is the same as that of the mud flow rate, which, from another aspect, further verifies that the data collected by the sensor are correct and can meet practical working conditions.

\section{Conclusions}

A new sensor for measuring the rotating speed of the downhole turbodrill is designed in consideration of practical conditions. This is a centrifugal force-based design scheme. Specifically, it is based on the functional relationship between the turbine speed and the centrifugal force. Besides, the sensor has been tested both in-lab and on-site. The test results show that the measurement error is not more than $\pm 4 \%$ which can meet the practical on-site conditions. However, this sensor still has some deficiencies that are required to be further improved:

(1) The friction coefficients of the sensor's materials have some effects on the accuracy of the measurement. In ideal conditions, the friction between the materials can remain the same. However, when the sensor has been used for a long period, properties of the materials will change (e.g., oxidation and rust) and their friction coefficients will also change accordingly, resulting in errors. Although we have made rustproof and antioxidation treatments, we still cannot completely keep material properties unchanged. Future work will be focused on strengthening material properties and improving rust-proof and antioxidation treatments to make the sensor more accurate and reliable for a long period.

(2) Due to the limitations of experiment conditions and research fund, we have only tested only six velocities in the lab. The six velocities dispersed in the measurement range of the sensor and, theoretically, can meet the requirements of testing the sensor. In the future, if we are supported with enough funding, we then have an option to use a stepless speed regulation device to make the data distribution better, and we 
can also modify and adjust each design parameter according to the test data to further improve the accuracy of the sensor.

\section{Conflicts of Interest}

The authors declare that they have no conflicts of interest.

\section{Acknowledgments}

This work was supported by the Fundamental Research Funds for the Central Universities, China University of Geosciences, Wuhan (CUG170655 and 1810491T06).

\section{References}

[1] S. L. Deng, "Research on deep prospecting problems and measures," World Nonferrous Metal, no. 1, pp. 205-206, 2017.

[2] C. Ojanomare, P. Cornetti, R. Romagnoli, and C. Surace, "Fatigue crack growth analysis of drill pipes during rotary drilling operations by the multiple reference state weight function approach," Engineering Failure Analysis, vol. 74, no. 4, pp. 1134, 2017.

[3] W. Yu, Y. Jianyi, and L. Zhijun, "Design and development of turbodrill blade used in crystallized section," The Scientific World Journal, vol. 2014, Article ID 682963, 12 pages, 2014.

[4] A. Mokaramian, V. Rasouli, and G. Cavanough, "Turbodrill design and performance analysis," Journal of Applied Fluid Mechanics, vol. 8, no. 3, pp. 377-390, 2015.

[5] A. Mokaramian, V. Rasouli, and G. Cavanough, "Fluid flow investigation through small turbodrill for optimal performance," Mechanical Engineering Research, vol. 3, no. 1, pp. 1-24, 2013.

[6] Z. Shi, Q. Huang, G.-S. Wu et al., "Design and development of a tachometer using magnetoelectric composite as magnetic field sensor," IEEE Transactions on Magnetics, vol. 99, pp. 15, 2017.

[7] B. R. Resor, M. W. Trethewey, and K. P. Maynard, "Compensation for encoder geometry and shaft speed variation in time interval torsional vibration measurement," Journal of Sound and Vibration, vol. 286, no. 4-5, pp. 897-920, 2005.

[8] X. Qinyang, The Design and Test of the Turbodrill's Stator, Southwest Petroleum University, Chengdu, 2015.

[9] H. Q. Liang, Z. W. Shao, and Q. Z. Chai, "Processing of turbodrill rotating speed signal with an adaptive noise canceller," in SPE Annual Technical Conference and Exhibition, p. 7, New Orleans, LA, USA, September 1990.

[10] C. Wu, G. Wen, L. Han, and X. Wu, "The development of a gas-liquid two-phase flow sensor applicable to CBM wellbore annulus," Sensors, vol. 16, no. 11, pp. 1943-1965, 2016.

[11] L. D. Zhao, L. X. Li, Y. Wang, Q. L. Xue, and B. L. Liu, "Summary of research progress in turbodrill," Exploration Engineering, vol. 43, no. 10, pp. 269-274, 2016. 


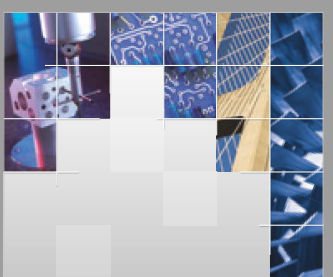

\section{Enfincering}
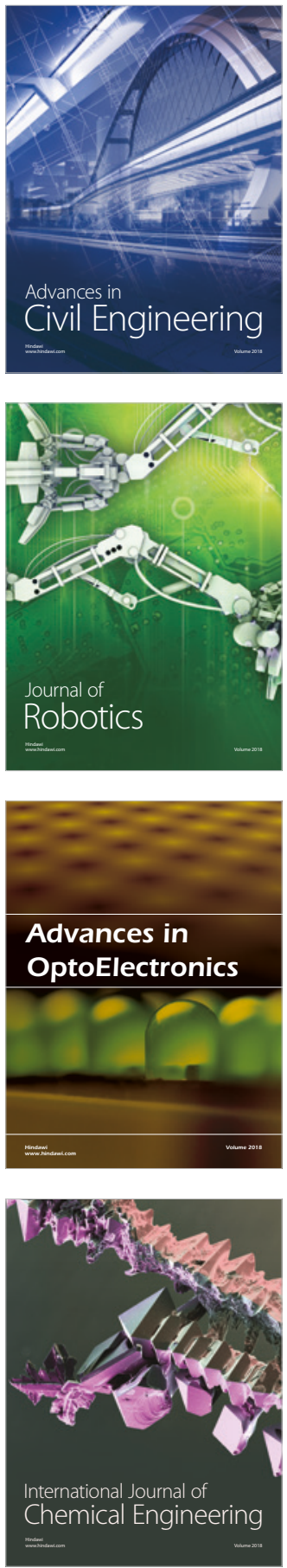

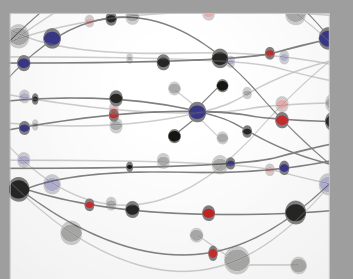

\section{Rotating \\ Machinery}

The Scientific World Journal

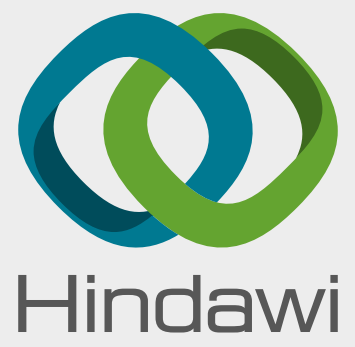

Submit your manuscripts at

www.hindawi.com
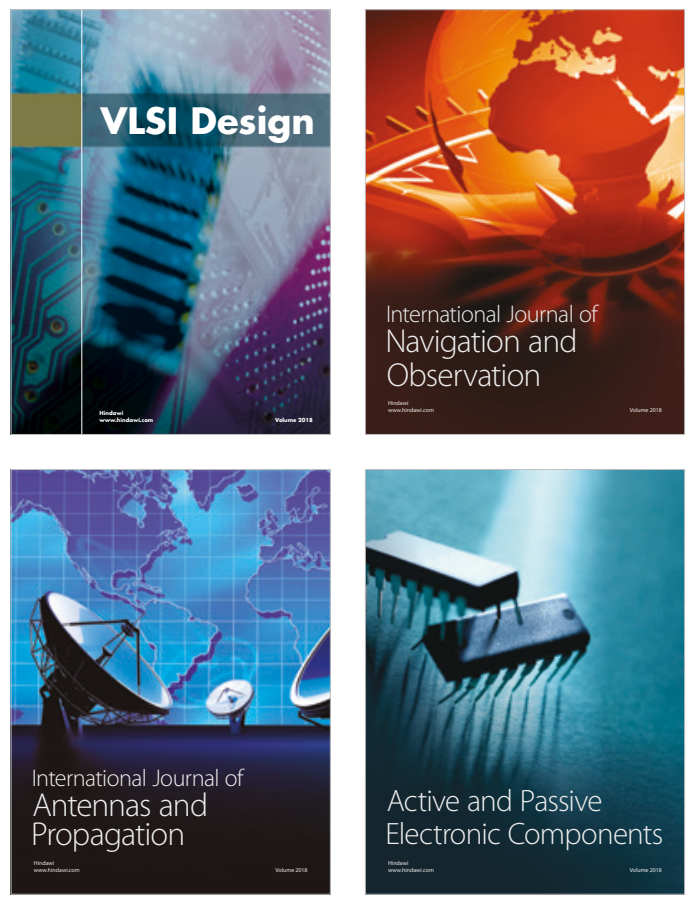
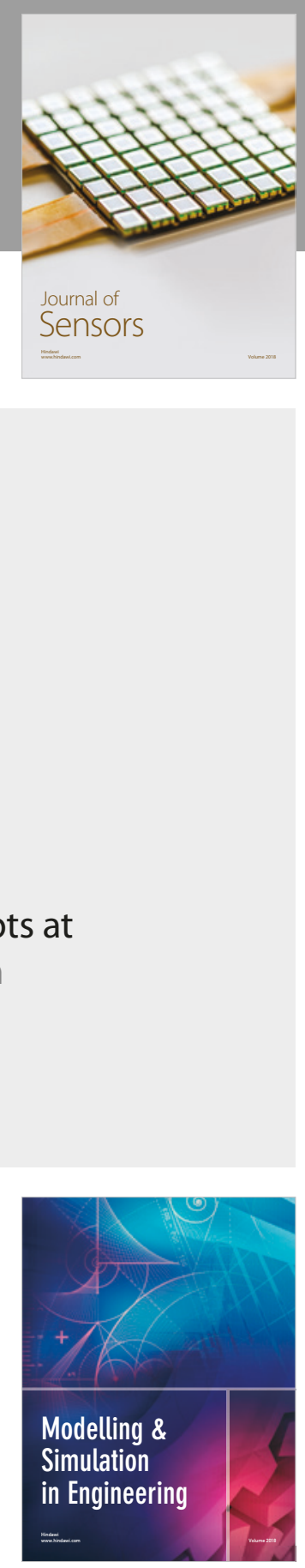

\section{Advances \\ Multimedia}
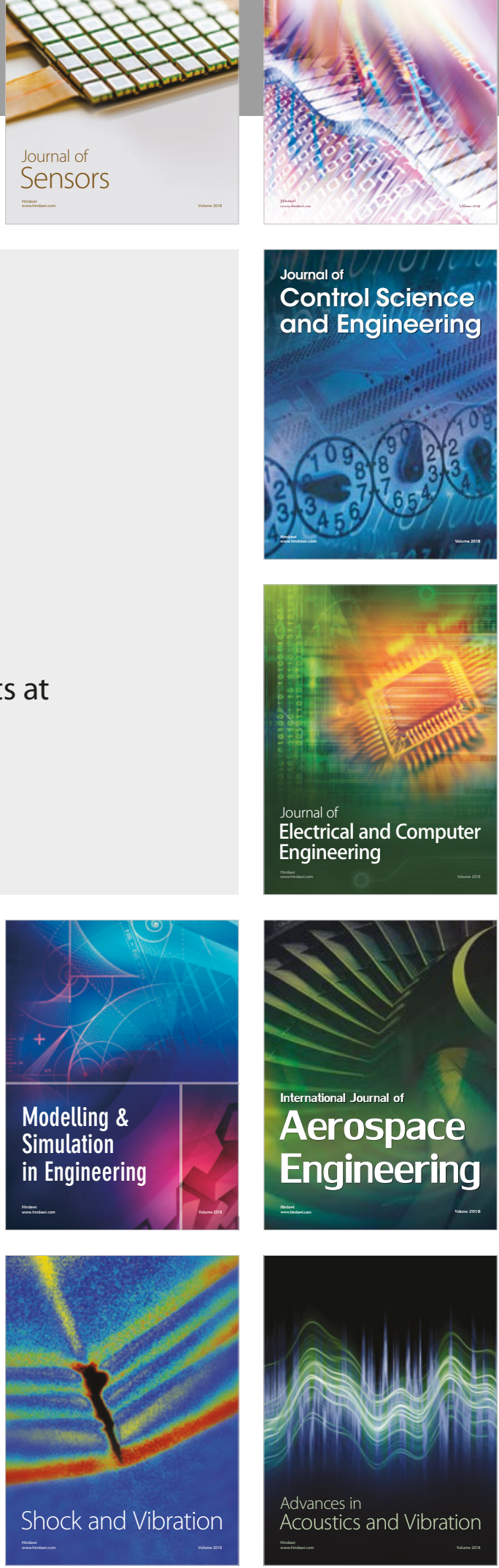